\title{
Estimation of Distribution System Reserve Capacity and Its Impact on System Reliability considering load growth
}

\author{
Galiveeti Hemakumar Reddy, Arup Kumar Goswami and Nalin B Dev Choudhury
}

Department of Electrical Engineering, National Institute of Technology Silchar, Assam, India

\begin{abstract}
The major objective of any electric distribution system (DS) operators in the present competitive market is to increase the service quality with improved reliability and lesser energy cost. This objective can be achieved by maintaining the balance between the reliability and cost of investments. In this work, system reliability is improved by using reserve capacity considering load growth. A sequential load growth model is presented for addition of load in non-integer years which is essential for sequential Monte Carlo simulation method. A model is presented to estimate the required reserve capacity if planning duration is known and vice versa. The concept of minimum allowable reserve capacity (MARC) is introduced to overcome the risk due to uncertain load growth. The system reserve capacity is increased by two ways i.e. up-gradation of distribution substation and integration of distributed generation (DG). The impact of load growth rate and reserve capacity level on system reliability is evaluated for a practical Indian distribution system. The required reserve capacity levels and capacity expansion duration are evaluated for different load growth rates and MARC values.
\end{abstract}

Keyword: Sequential load growth, reserve capacity, capacity expansion, reliability, distributed generation.

\section{Introduction}

In the present competitive deregulated environment, the distribution system (DS) operators are under high pressure to improve the system reliability, reduce the energy cost and increase the profit simultaneously. The deregulation of energy market brings in the concept of performance based rates (PBR), which is a contract of reward/penalty for system operators based on the system performance i.e. reliability. However, the PBR introduces the financial risk to the system operators [1] i.e. imposing penalty for not maintaining predefined reliability levels. To reduce the financial risk associated with the use of PBR, proper estimation of future system reliability, planning of system capacity expansion and maintaining appropriate reserve capacity are necessary.

In the literature, different reliability evaluation methods are developed for distribution system reliability evaluation. In reference [2], reliability of a test system is evaluated using analytical approach and the results considered as benchmark. The analytical methods are fail to consider the uncertainty involved in the assessment due to uncertainty of system failures and repair times. Monte Carlo simulation (MCS) methods are used for reliability evaluation [3], [4] to overcome the disadvantage of analytical method. The MCS methods has the ability to include the uncertainty associated with the distributed generators (DGs) output power. System reliability is evaluated in [5], [6] with the integration of DGs. The computational efficiency of MCS method is less and takes more time for evaluation. Authors in [7], [8] are used variance reduction techniques in association with MCS method to improve the computational efficiency of MCS method.

The loading of the system is not constant and it increases with time due to load growth. Reliability of a system is not authenticate if load growth is not included in the evaluation. The effects of load growth on different distribution system parameters are discussed in [9] - [11]. The uncertainty in load growth is modelled using fuzzy concept [12], [13] while probability load growth is considered [14] to find the supply capacity of the DS. The existing load growth models discussed in [9] -[14] considered the time period in integer values in years, which adds the

Received: October $10^{\text {th }}, 2017$. Accepted: December $27^{\text {th }}, 2017$ 
additional load due to the load growth on annual basis. However, in practical scenario, additional loads are added to the system throughout the year. Hence, existing load growth models fail to add the additional load to the system throughout the year. Therefore, they are not applicable in sequential evaluation of system reliability as such evaluation requires loading of the system at any instant in a year.

As the load growth increases the system demand in the future, the DS operators need to go for their capacity expansion. The extra added capacity is technically called as reserve capacity of the system at the initial stage. The estimation of the required reserve capacity plays a key role in capacity expansion planning. The reserve capacity increases by the means of integration of DGs and reinforcement or installation of distribution substation and feeders [15]. In reference [16], the generation reserve capacity forecasting is done using second order Markov chain and Monte Carlo simulation. In [17], the reserve capacity is estimated by considering energy charging and customers' contribution. Keeping the reserve capacity not only satisfies the future loads of the system due to load growth but also increases the reliability of system. The impact of reserve margin on system reliability and economic operation is studied in [18].

The assessment of realistic reliability demands the consideration of appropriate load growth model. The model will be suitable for calculating system maximum load for non-integer years, which needs to add the additional load to the system on monthly basis, so that model is more suitable to use with sequential MCS for reliability evaluation. The over investment for capacity expansion is economically not good for system operation and insufficient reserve capacity will not only create the power shortage in the system but reduce the reliability as well. For estimation of reserve capacity certain load growth rate is required but as the load growth rate is uncertain in nature [12] - [14], this uncertain growth rate increases the risk of insufficient reserve capacity. Hence, an additional margin of reserve capacity is needed to mitigate the risk.

Therefore in this paper a sequential load growth model is proposed to overcome the drawbacks of existing load growth models. Models are proposed to estimate the required reserve capacity and required time for next capacity expansion considering the system load growth. The risk of uncertain load growth rate is mitigated using an extra reserve capacity called as Minimum Allowable Reserve Capacity (MARC).

The remaining paper is organised as follows: The introduction of reliability evaluation is explained in section 2, which includes component modelling, load modelling and description of sequential MCS method. The mathematical modelling of sequential load growth model and estimation of reserve capacity is formulated in section 3. The proposed approach for impact analysis is explained in section 4 with load growth and reserve capacity. The test distribution system description and reliability data are mentioned in section 5. Results are analysed and relevant discussion are forwarded in section 6 . Finally section 7 concludes the work.

\section{Brief introduction to reliability evaluation}

\section{A. Component modelling}

The component modelling is very essential for system reliability evaluation. Typically, distribution system components (transformers, circuit breakers, bus bars, line sections, switching devices etc.) have two states i.e. operating and failure state. These two states are modelled using continuous time Markov modelling to represent the component reliability model [19], [20]. The state transition rate depends on the failure and repair rates of the component.

The state space representation of two state continuous time Markov model is shown in Figure 1. The component operating and failure states are represented by up and down states respectively. For sequential MCS method, the artificial failure and repair times are generated by using exponential probability distributions [20].

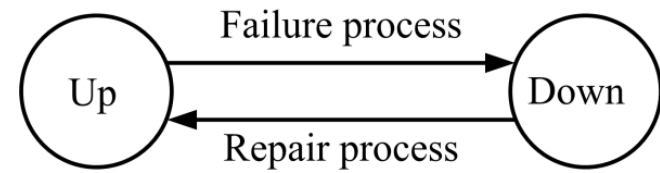

Figure 1. Two state space diagram of the component 


\section{B. Load modelling}

The DS loading depends on many aspects like season (rainy, summer and winter), social factors (major festivals, marriages etc.) and weather conditions etc. The load changes of the DS have a repeated nature of daily and monthly basis. The time varying load models will give the realistic representation of system loading. The system loading at any instant $t$ is determined by using the following equation [21].

$$
P(t)=W_{h}(t) \times \mathrm{W}_{m}(m) \times P_{\max }
$$

where,

$W_{h}(t)=$ Hourly load factor

$\mathrm{W}_{m}(m)=$ Monthly load factor

$P(t)=$ System loading at time $t$

$P_{\max }=$ System maximum loading

$m=$ month index

The hourly load factor is the percentage of load at a particular hour in terms of daily peak load. The monthly load factor is the percentage of maximum load in a month in terms of annual peak load.

\section{Reliability indices}

The distribution system reliability is measured using system reliability indices i.e. system average interruption frequency index (SAIFI), system average interruption duration index (SAIDI), customer average interruption duration index (CAIDI), expected energy not supplied (EENS) and average service availability index (ASAI) etc. System reliability indices are determined by using load point reliability indices like failure rate and annual outage time. The reliability indices are calculated as follows [19]:

$$
\begin{aligned}
& \text { SAIFI }=\frac{\sum \lambda_{i} N_{i}}{\sum N_{i}} \\
& \text { SAIDI }=\frac{\sum U_{i} N_{i}}{\sum N_{i}} \\
& \text { CAIDI }=\frac{\sum U_{i} N_{i}}{\sum \lambda_{i} N_{i}} \\
& \text { EENS }=\frac{\sum L_{i} U_{i}}{\sum S A I=\frac{\sum N_{i} \times 8760-\sum U_{i} N_{i}}{\sum N_{i} \times 8760}}
\end{aligned}
$$

Where $\lambda_{i}$ is the failure rate, $U_{i}$ is the annual outage time, $N_{i}$ is the number of customers, $L_{i}$ is the load of load point $i$.

\section{Sequential MCS}

Simulation methods are intended to study and calculate the real behaviour of the system during the analysis period. The evaluation process is completely based on the generation of random numbers because it reflects random nature of system behaviour. Sequential MCS method is able to use the time varying loads for reliability evaluation of the DS. The basic features of the 
sequential MCS method with variance reduction techniques are used to evaluate the system reliability in this paper. The simulation process is stopped when variation of reliability indices are less than the predefined coefficient of variation $(\beta)$. Variance reduction techniques will reduce computational time of MCS method. In present work, Antithetic variates is used as a variance reduction technique [7]. The reliability indices are estimated using sequential MCS method. The mean of the indices over $N$ simulation years is determined by [8]:

$$
\tilde{E}[F]=\frac{1}{N} \sum_{k=1}^{N} F\left(x^{k}\right)
$$

here, $F$ is the function definition of reliability index. $x^{k}$ is the sequence of system states in simulation year $k$. The variance of estimator is calculated as follows:

$$
V(\tilde{E}[F])=\frac{V(F)}{N}
$$

here, $V(F)$ is variance of function.

The coefficient of variance is determined by

$$
\beta=\frac{\sqrt{V(\tilde{E}[F])}}{\tilde{E}[F]}
$$

Proposed load growth and reserve capacity estimation modelling

\section{A. Load growth modelling}

Load growth adds the energy demand on the distribution system. Increase of energy demand is caused by many social and economic reasons (population growth, industrialization, changing the lifestyle of consumers etc.). Loading of the system under the load growth condition is determined as follows [12]:

$$
P_{n}=P_{i}(1+r)^{n}
$$

where,

$P_{i}=$ Initial or present load of the system

$P_{n}=$ Final or maximum load of the system after $n$ years

$n=$ Time period in years $(n=1,2,3 \ldots)$

$r=$ Load growth rate per year

If final load and load growth rate are known values then time required to reach the final load is determined as follows:

Applying logarithmic on both sides of eq. (10)

$$
\begin{aligned}
& \log P_{n}=\log \left(P_{i}(1+r)^{n}\right) \\
& \log P_{n}=\log P_{i}+n \log (1+r)
\end{aligned}
$$

From eq (12), time period $n$ is calculated as:

$$
n=\frac{\log P_{n}-\log P_{i}}{\log (1+r)}=\log _{(1+r)}\left(P_{n} / P_{i}\right)
$$

The load growth model represented in eq. (10) is a non-sequential growth model which takes the addition of load on a yearly basis instead of considering the addition throughout the year and hence it is not feasible to use with sequential MCS. To overcome this drawback, a sequential load growth model is proposed in this paper which gives the increment of the load on the system in coordination with sequential MCS method. The modelling of sequential load growth model is developed as follows: 
Let, $x$ be the load growth rate per month. The final load after $k$ months is determined by

$$
P_{n}=P_{i}(1+x)^{k}
$$

If time period is one year then $n=1$ and $k=12$. Then $n$ and $k$ values are substituted in eq. (10) and eq. (14)

$$
\begin{aligned}
& P_{n}=P_{i}(1+x)^{12} \\
& P_{n}=P_{i}(1+r)
\end{aligned}
$$

From eq. (15) and eq. (16)

$$
(1+x)^{12}=1+r
$$

Applying $12^{\text {th }}$ root on both sides of eq. (17) and it becomes

$$
1+x=(1+r)^{1 / 12}
$$

1Substitute eq. (18) in eq. (14) to find the system final load

$$
P_{n}=P_{i}(1+r)^{k / 12}
$$

If the time period is more than 12 month i.e. $k>12$ then time period is combination of years and months. Equation (19) becomes

$$
P_{n}=P_{i}(1+r)^{q}
$$

where, $q=N+\frac{m}{12}$

$m=$ Time duration in months $(m=1,2, \ldots .12)$

$N=$ Time duration in years $(N=0,1,2 \ldots, n-1)$

\section{B. System reserve capacity estimation}

System reserve capacity is helpful to partial or full restoration of affected customers after the isolation of faulted section. The system restoration capacity will increase by keeping more reserve capacity. The system operators need to estimate the required reserve capacity for reliable operation of the system during a particular time period and required time for next capacity expansion is based on the present available reserve capacity. The mathematical modelling of estimation of reserve capacity and time required for capacity expansion is as follows.

The maximum loading capacity $\left(P_{\max }\right)$ of the system with inclusion of reserve capacity $(R C)$ is calculated as follows.

$$
P_{\max }=(1+R C) P_{i}
$$

Final load at the end of time period $q$ with a load growth rate $r$ is determined from the eq. (20) and is also called as maximum loading capacity $\left(P_{\max }\right)$ required at the end of time period $q$.

$$
P_{\max }=P_{i}(1+r)^{q}
$$

As mentioned in the introduction, the load growth rate is not a fixed value and have uncertain nature. The system loading may reach to its maximum loading capacity before the end of the time period $q$. Thus might fail to maintain the system reliability as desired.

Hence, from the reliability perspective of the system operation, it is mandatory that the system must have a certain amount of reserve capacity at any instant or at the end of planning period to overcome the impact of uncertainty in load growth rate. This mandatory reserve capacity is called as minimum allowable reserve capacity (MARC). Once the system reserve capacity is reached to $M A R C$ level, then the system must undergo for capacity expansion. So the 
maximum loading capacity of the system at the end of period $q$ while maintaining a desired $M A R C$ level is given below.

$$
P_{\max }=P_{i}(1+M A R C)(1+r)^{q}
$$

From eq. (21) and eq. (23)

$$
(1+R C)=(1+M A R C)(1+r)^{q}
$$

In eq. (24), $M A R C$ and $r$ are known parameters and $R C$ and $q$ are unknown parameters. If the capacity expansion period $q$ is known, then the required reserve capacity $R C$ is calculated using the following equation.

$$
R C=(1+M A R C)(1+r)^{q}-1
$$

If the available reserve capacity $R C$ is known, then the time required for system capacity expansion is formulated as follows:

Applying logarithmic on both sides of eq. (24)

$$
\begin{aligned}
& \log (1+R C)=\log (1+M A R C)+q \log (1+r) \\
& q=\frac{\log (1+R C)-\log (1+M A R C)}{\log (1+r)} \\
& q=\log _{R} K
\end{aligned}
$$

where, $K=\frac{1+R C}{1+M A R C}$ and $R=1+r$

\section{Reserve capacity installation}

Two scenarios are considered to increase the system reserve capacity. Scenario 1 considers the increase of substation capacity and up gradation of feeders. This will increase the availability of the substation and load transfer capability of feeders. Scenario 2 considers the integration of DGs without changing the substation and feeders capacity. This will increase the islanding capacity and also the load transfer capacity of feeders. The correlation between feeder loading and DG output are considered in reliability assessment. For this purpose, time varying loads [7] and DG output models are considered [22]. The change of feeder loading on substation in scenario 1 is as follows.

$$
P_{F_{-} \text {new }}(t)=P_{F_{-} \text {old }}(t)+P_{L G}(t)
$$

where, $P_{F_{-} \text {old }}(t)$ is initial feeder loading. $P_{L G}(t)$ is the increase of load due to load growth. $P_{F_{-} \text {new }}(t)$ is the final or new feeder loading.

The change of feeder loading on substation in scenario 2 is as follows:

$$
P_{F_{-} \text {new }}(t)=P_{F_{-} \text {old }}(t)+P_{L G}(t)-P_{D G_{-} \text {gen }}(t)
$$

Here, $P_{D G_{-} g e n}(t)$ is DG output power.

It is to be noted that the load transfer capacity of any feeder depends on maximum loading capacity of feeder and present loading of feeder.

\section{Frame work for reliability evaluation considering load growth and reserve capacity}

The proposed work in this paper uses the basic features of sequential MCS methods for reliability evaluation. But it considers all components and normal working conditions of a 
practical system. The load transfer restrictions are applied and load transfer capacity of the feeders varies according to the instantaneous loading of the system. The time varying loads are applied to reliability evaluation with the help of hourly and monthly load factors. Two different scenarios are considered to increase the reserve capacity of the system. The load growth and estimation of reserve capacity models are integrated with sequential MCS method for accurate estimation of system reliability. The proposed sequential load growth model gives the realistic load addition suitable for sequential evaluation. The inclusion of MARC for reserve capacity estimation reduces the risk of uncertain load growth as discussed in previous sections. The system instant loading after adding the additional load is determined in association with TTF of system equipment. The feeders load transfer capability is re-evaluated considering the MARC, load growth rate and TTF. Different load growth rates, reserve capacity levels and MARC values are used for evaluation purpose to analyse their impact on reliability. The evaluation steps of proposed approach are represented as a flow chart as shown in Figure 2.

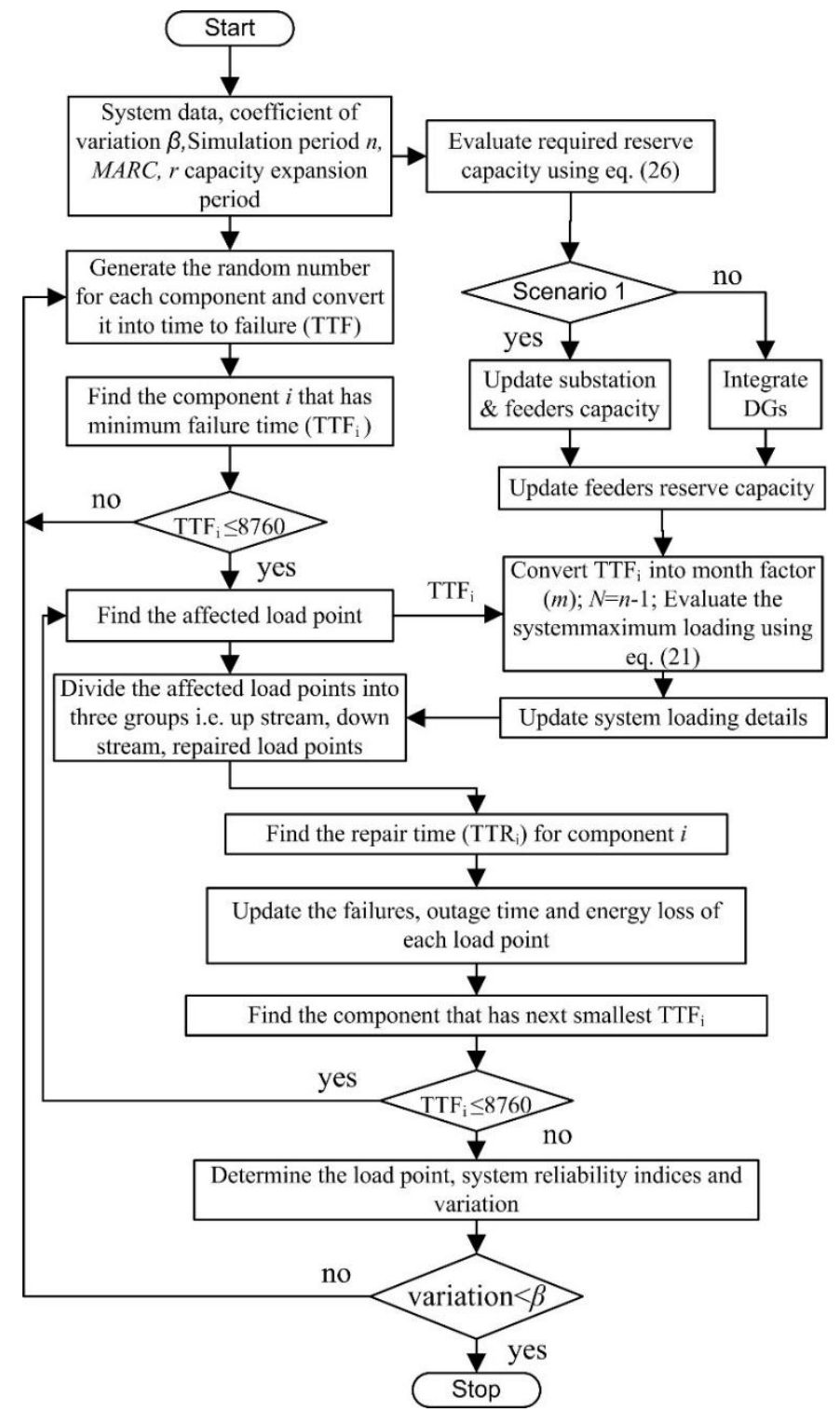

Figure 2. Flow chart for reliability evaluation using MCS method 


\section{Test system}

An Indian distribution system is used as a test system as given in Figure 3. It is a radially operated and lightly interconnected system which uses normally open (NO) switches to transfer loads between the feeders during the faults. The required data for reliability analysis has been collected from a practical system of Assam Power Distribution Corporation Limited. The system loading data is used to develop the time varying load models. The distribution substation having 25 MVA installed capacity with three power transformers (rated at 10 MVA, 10 MVA and 5 MVA) are given in Figure 4. At present, the maximum load on the distribution system is $18 \mathrm{MW}$ (at 0.8 p.f lagging) which experiences a $10 \%$ load growth rate per year. The detailed layout and connection of major substation equipment is shown in Figure 4. This distribution system has 8 feeders and 295 load points. The load points are distributed along the feeders and serves 6440 customers of different types (which includes large, commercial and domestic customers). Feeders are divided into 59 sections using sectionalizing switches to reduce the affected customers during the fault. Six NO switches (namely SW1-SW6) are used to interconnect the system for load transfer during the failures. The locations of switches are shown in Figure 3. The failure rate $(\lambda)$ and repair $(r) /$ replacement time $\left(r_{p}\right)$ of the system components are given in Table 1.

Table 1 . Test system reliability data

\begin{tabular}{lcc}
\hline \multicolumn{1}{c}{ Component } & $\begin{array}{c}\text { Failure rate } \\
\text { (failures/year) }\end{array}$ & $\begin{array}{c}\text { Repair/replacement } \\
\text { time (hours) }\end{array}$ \\
\hline Distribution transformer & 0.0843 & 10 \\
Power transformer & 0.0843 & 15 \\
Overhead lines & 0.1479 & 5 \\
Circuit breaker & 0.004 & 4 \\
Bus bar & 0.0001 & 2 \\
\hline
\end{tabular}

The repair of transformers takes more time, so it is considered that the failed transformer is replaced with a healthy (spare) transformer, unlike the on-site repair of other equipment (circuit breakers, bus bars, line). Initially the distribution substation has a $10 \%$ reserve capacity (at 0.8 p.f. lagging) and feeders have sufficient reserve capacity to handle future load growth of the system. For SC 1, system reserve capacity is increased by considering the following assumptions: System power factor is increased to 0.9 p.f lagging using compensation devices and replacing the 5 MVA power transformer (T3) by 10 MVA. DGs are installed to increase the reserve capacity of the system for SC 2. The DG locations are shown in Figure 3. The size of the DG are changed according to the required reserve capacity.

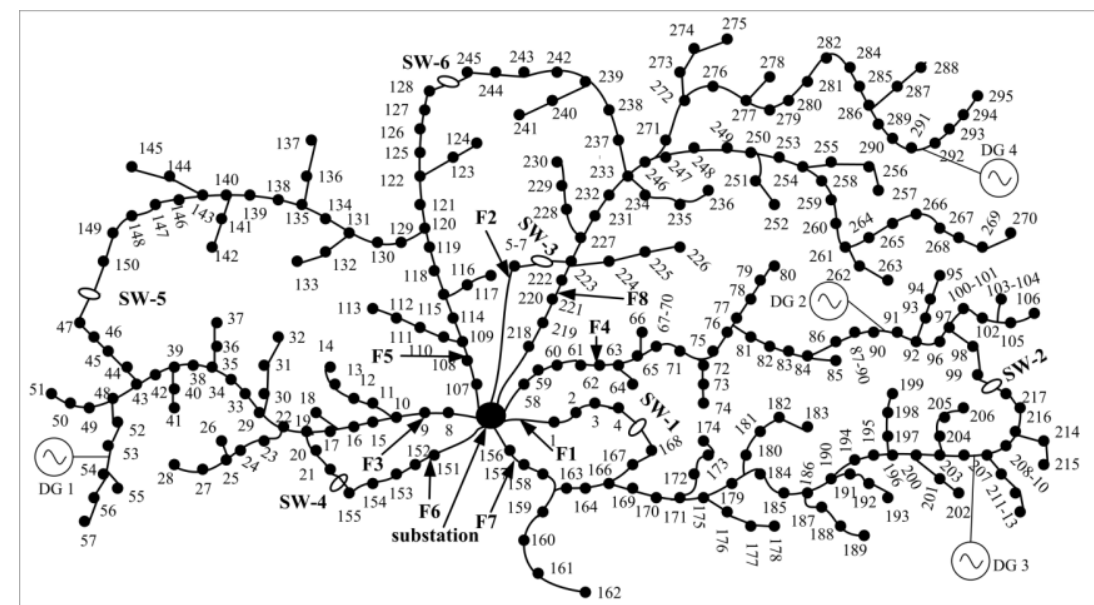

Figure 3. Single line diagram of the test distribution system 


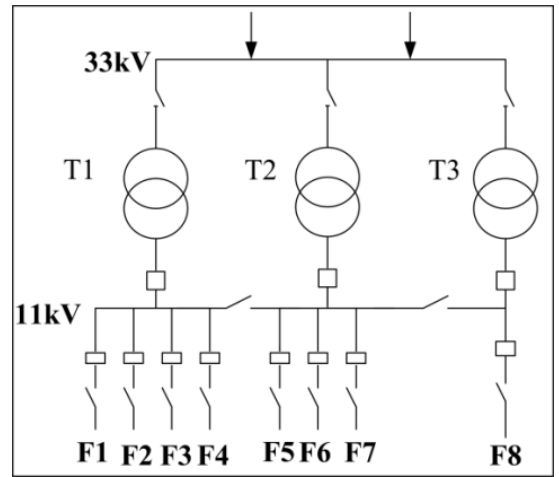

Figure 4. Single line diagram of the test distribution substation

\section{Results and discussions}

The paper utilises the practical Indian distribution system, as discussed previously, for analysis purpose. It is assumed that the system will be operated manually and interconnected through NO switches for all the analysis done in this section. The presented results in this section explains load growth and reserve capacity impact on system reliability. Here, reserve capacity means the reserve capacity of both substation and the feeders reserve capacity. The load growth is occurred due to two reasons, i). New customers are added to the system ii). Extra loads are added by the existing customers. It is assumed that number of load points remain same during the analysis period.

\section{A. Impact of load growth and reserve capacity}

System reliability is calculated for different load growth rates and reserve capacities. Three different cases are studied to evaluate the impact of load growth and reserve capacity on system reliability and obtained results are compared with each other. The basic system reliability indices viz. SAIFI (interruptions/year), SAIDI (hours/year), CAIDI (hours/year), EENS (MWhr/year) and ASAI are determined for comparison.

CASE 1: In this case, the system reliability is calculated without system load growth. The system reliability indices (SAIFI, SAIDI, CAIDI, EENS and ASAI) are calculated by changing the reserve capacity from 0 to $70 \%$, so that the impact of reserve capacity on reliability is identified.

CASE 2: In this case, the system reliability is calculated by considering the system load growth. The evaluation of the distribution system final load is determined using load growth model given in eq. (10).

CASE 3: In this case, the proposed sequential load growth model is used for determination of system final load. This model is able to give the instantaneous system loading in coordination with the sequential MCS method for reliability calculation, the mathematical model for which is given in eq. (21).

Scenario 1 is considered for increase of reserve capacity and impact of load growth rate and reserve capacity is analysed in the following section. The system reliability indices for different levels of reserve capacity are shown in Figure 5 for case 1. From the Figure 5, it is observed that SAIFI is not affected due to the change of system reserve capacity. It is expected because the SAIFI value depends on failure rate and number of customers in each load point. System reserve capacity increases the restoration capacity and reduces the restoration time/outage time of load points and it will not show any impact on failure rate. On the other hand, SAIDI, CAIDI and EENS indices depends on the restoration/outage time of the load points. These reliability indices 
are changing with change of system reserve capacity and improves with increase of reserve capacity.

EENS is significantly improved, if the system reserve capacity changes from $0 \%$ to $10 \%$ and from $10 \%$ to $20 \%$. There is a small improvement in EENS, if system reserve capacity changes from $20 \%$ to $30 \%$ and from $30 \%$ to $40 \%$. It is also identified that there is a big improvement in SAIDI and CAIDI values, if the reserve capacity increases from $0 \%$ to $20 \%$ and small variation between $20 \%$ to $40 \%$ reserve capacities. If system reserve capacity increases further (more than $40 \%$ ) then relative improvement of SAIDI, CAIDI and EENS is very less and negligible. This is explained as, if feeders don't have reserve capacity then loads are not restored after isolation of faulty section. However, if the feeders reserve capacity is increased from $0 \%$ to $20 \%$, it is but obvious to have more number of load point getting restored after isolation of faulty section. Because of this the relative improvement of reliability is high. Now, if the reserve capacity goes on increasing then relatively less number of load points would be restored which causes less improvement in reliability. After a certain level of reserve capacity (40\%), negligible load points are restored and consequently the relative improvement in reliability is also negligible. It is also observed that from the reliability point of view, the minimum required reserve capacity is between $20-40 \%$ for this case study.

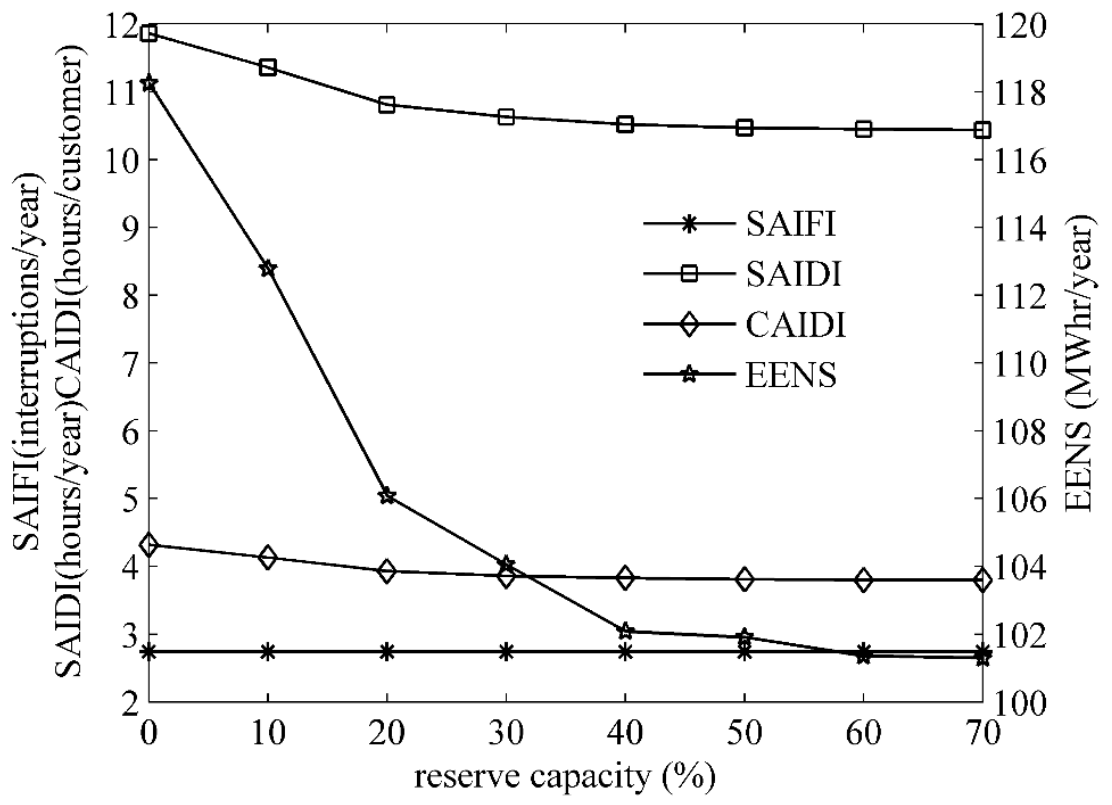

Figure 5. System reliability indices without load growth

The system reliability indices are calculated for case 2 for different reserve capacity levels (from $20 \%$ to $70 \%$ ) and load growth rates $(5 \%, 10 \%, 15 \%$, and $20 \%$ ). The system reliability values are given in Table 2. From Table 2, it is observed that apart from the reliability improvement due to increase of reserve capacity, the system reliability reduces with the increase of the system load growth rate. It is because of the following reasons: reserve capacity will increase the load transfer capacity of the feeders but at the same time due to load growth, loading of all feeders increases and it leads to reduction of load transfer capacity of the feeders. If load growth rate is fixed then reliability is improving with increase of reserve capacity and reasons are stated above. If reserve capacity is fixed then system reliability is decreasing with increase of load growth rate. It can be seen from Table 2 that there is no further considerable improvement of reliability beyond $50 \%$ reserve capacity for $5 \%$ load growth rate whereas the reliability is seen to be improved for $20 \%$ load growth rate till the reserve capacity of $70 \%$. Hence, in conclusion the minimum required reserve capacity increases with the increase of load growth rate. 
Estimation of Distribution System Reserve Capacity and Its Impact

Table 2. System reliability indices for case 2

\begin{tabular}{|c|c|c|c|c|c|c|c|}
\hline \multirow{2}{*}{ Index } & \multirow{2}{*}{ Growth rate } & \multicolumn{6}{|c|}{ Reserve capacity } \\
\hline & & $20 \%$ & $30 \%$ & $40 \%$ & $50 \%$ & $60 \%$ & $70 \%$ \\
\hline \multirow{6}{*}{ EENS } & $5 \%$ & 111.63 & 109.98 & 108.83 & 107.35 & 106.76 & 106.35 \\
\hline & $10 \%$ & 118.84 & 116.15 & 114.72 & 112.90 & 112.23 & 111.68 \\
\hline & $15 \%$ & 125.99 & 122.99 & 120.84 & 118.87 & 117.85 & 117.10 \\
\hline & $20 \%$ & 133.42 & 129.93 & 127.41 & 125.86 & 123.52 & 122.25 \\
\hline & $5 \%$ & 10.85 & 10.67 & 10.58 & 10.49 & 10.46 & 10.44 \\
\hline & $10 \%$ & 10.93 & 10.72 & 10.63 & 10.52 & 10.48 & 10.45 \\
\hline \multirow{2}{*}{ SAIDI } & $15 \%$ & 11.050 & 10.82 & 10.68 & 10.56 & 10.51 & 10.46 \\
\hline & $20 \%$ & 11.19 & 10.93 & 10.76 & 10.61 & 10.54 & 10.49 \\
\hline \multirow{6}{*}{ CAIDI } & $5 \%$ & 3.9598 & 3.8941 & 3.8613 & 3.8284 & 3.8175 & 3.8102 \\
\hline & $10 \%$ & 3.9890 & 3.9124 & 3.8795 & 3.8394 & 3.8248 & 3.8138 \\
\hline & $15 \%$ & 4.0328 & 3.9489 & 3.8978 & 3.8540 & 3.8357 & 3.8175 \\
\hline & $20 \%$ & 4.0839 & 3.9890 & 3.9270 & 3.8722 & 3.8467 & 3.8284 \\
\hline & $5 \%$ & 0.998761 & 0.998781 & 0.998792 & 0.998802 & 0.998805 & 0.998808 \\
\hline & $10 \%$ & 0.998752 & 0.998776 & 0.998786 & 0.998799 & 0.998803 & 0.998807 \\
\hline \multirow{2}{*}{ ASAI } & $15 \%$ & 0.998738 & 0.998764 & 0.998780 & 0.998794 & 0.998800 & 0.998805 \\
\hline & $20 \%$ & 0.998722 & 0.998752 & 0.998771 & 0.998788 & 0.998796 & 0.998802 \\
\hline
\end{tabular}

The system reliability values for case 3 are given in Table 3 for different load growth rates and reserve capacity levels. Variation of reliability indices with reserve capacity and load growth rate is same as that of case 2 however, the reliability is observed to be improved when compared to case 2 . For example, at $40 \%$ reserve capacity and $10 \%$ load growth, EENS is increased by $11.65 \%$ and $3.19 \%$ in case 2 and case 3 respectively as compared to case 1 . It is because the load growth model used in case 2 calculates system final load at the end of the year. It means that total load growth of the system during the simulation year is added at the starting of the simulation year. However, in general, total load growth will not add at starting of the simulation year but will add to system during entire simulation year. Due to this, the sequential reliability evaluation takes apparently more system loading than actual loading which reduces the net reserve capacity and there upon reliability changes accordingly (increase in reliability values than actual). The growth model used in case 3 calculates the final load at the ending of the each month of simulation year. The system maximum load will change according to the month of the year and this will match the actual system. Because of this reason, reliability values determined in case 3 are more realistic and lower than case 2.

Table 3. System reliability indices for case 3

\begin{tabular}{|c|c|c|c|c|c|c|c|}
\hline \multirow{2}{*}{ Index } & \multirow{2}{*}{ Growth rate } & \multicolumn{6}{|c|}{ Reserve capacity } \\
\hline & & $20 \%$ & $30 \%$ & $40 \%$ & $50 \%$ & $60 \%$ & $70 \%$ \\
\hline \multirow{6}{*}{ EENS } & $5 \%$ & 106.85 & 105.21 & 104.13 & 103.07 & 102.56 & 102.22 \\
\hline & $10 \%$ & 108.52 & 106.73 & 105.55 & 104.30 & 103.67 & 103.26 \\
\hline & $15 \%$ & 110.01 & 108.19 & 106.84 & 105.46 & 104.75 & 104.43 \\
\hline & $20 \%$ & 111.72 & 109.69 & 108.21 & 106.75 & 105.90 & 105.45 \\
\hline & $5 \%$ & 10.81 & 10.64 & 10.54 & 10.48 & 10.45 & 10.43 \\
\hline & $10 \%$ & 10.83 & 10.65 & 10.56 & 10.49 & 10.46 & 10.43 \\
\hline \multirow{2}{*}{ SAIDI } & $15 \%$ & 10.84 & 10.68 & 10.58 & 10.5 & 10.46 & 10.45 \\
\hline & $20 \%$ & 10.86 & 10.71 & 10.60 & 10.52 & 10.47 & 10.45 \\
\hline \multirow{6}{*}{ CAIDI } & $5 \%$ & 3.9452 & 3.8832 & 3.8467 & 3.8248 & 3.8138 & 3.8065 \\
\hline & $10 \%$ & 3.9525 & 3.8868 & 3.8540 & 3.8284 & 3.8175 & 3.8065 \\
\hline & $15 \%$ & 3.956 & 3.8978 & 3.8613 & 3.8321 & 3.8175 & 3.8138 \\
\hline & $20 \%$ & 3.9635 & 3.9087 & 3.8686 & 3.8394 & 3.8211 & 3.8138 \\
\hline & $5 \%$ & 0.998761 & 0.998781 & 0.998792 & 0.998802 & 0.998805 & 0.998808 \\
\hline & $10 \%$ & 0.998752 & 0.998776 & 0.998786 & 0.998799 & 0.998803 & 0.998807 \\
\hline \multirow{2}{*}{ ASAI } & $15 \%$ & 0.998738 & 0.998764 & 0.998780 & 0.998794 & 0.998800 & 0.998805 \\
\hline & $20 \%$ & 0.998722 & 0.998752 & 0.998771 & 0.998788 & 0.998796 & 0.998802 \\
\hline
\end{tabular}

The reliability values from all three cases are compared to analyse the impact of reserve capacity as well as load growth models. For the comparison, the load growth rate is assumed as $10 \%$. The comparison of EENS and SAIDI values are shown in Figure 6 and Figure 7 respectively for different reserve capacity levels. The EENS and SAIDI values are decreasing 
with the increase of reserve capacity for all the three cases. It is also observed that reliability indices in case 3 are less affected with system load growth as compared to case 2 for any level of reserve capacity and also at higher reserve capacity levels (above 50\%), the impact of load growth rate on SAIDI is observed to be very less in case 3 .

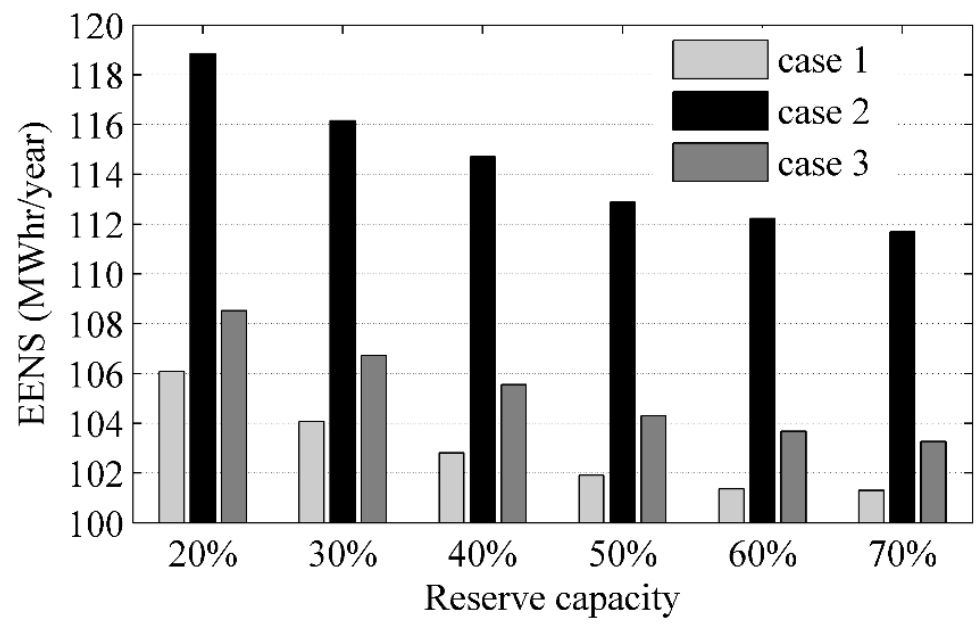

Figure 6. EENS value for different reserve capacity levels

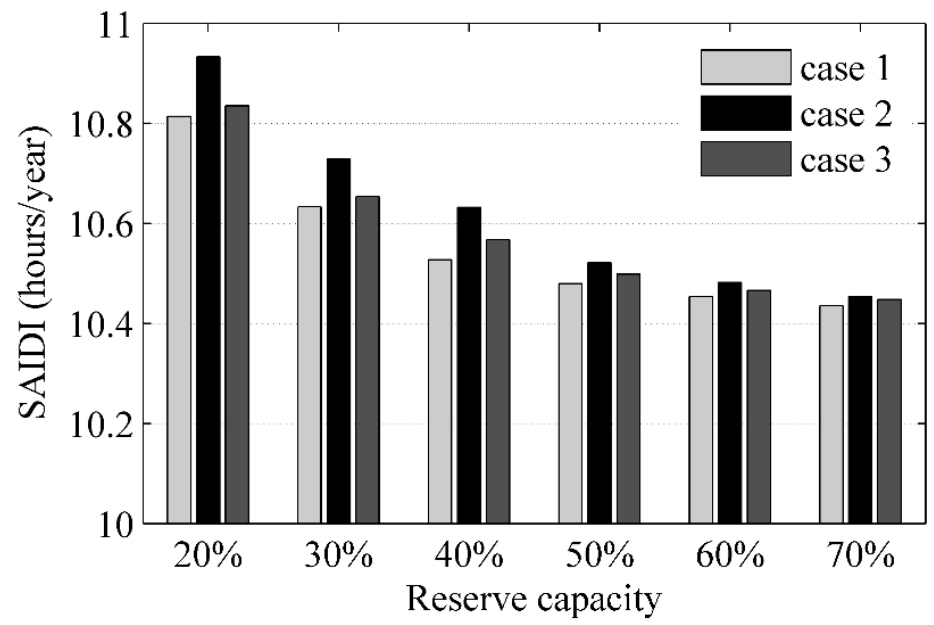

Figure 7. SAIDI value for different reserve capacity levels

The system reliability indices for different simulation years are shown in Figure 8. Figure 8 compares the reliability indices of case 2 and case 3 . The system reserve capacity is assumed as $60 \%$ at the starting of first simulation year and $10 \%$ load growth rate is assumed for all simulation years. It is observed that there is a slight increment of SAIDI value with increase of simulation year for both the cases. On the other hand, higher increment in EENS value is identified. The SAIDI and EENS values in simulation year 4 are increased by $3.05 \%$ and $39.37 \%$ respectively as compared to the values of simulation year 1 . The SAIFI value is constant and independent of the simulation year. SAIFI value mainly depends on the failure rate of load points and load point failure rate will depend on the failure rate of the distribution system equipment. The failure rate of distribution system equipment is constant throughout the analysis period and aging effect on equipment failures is not considered. 


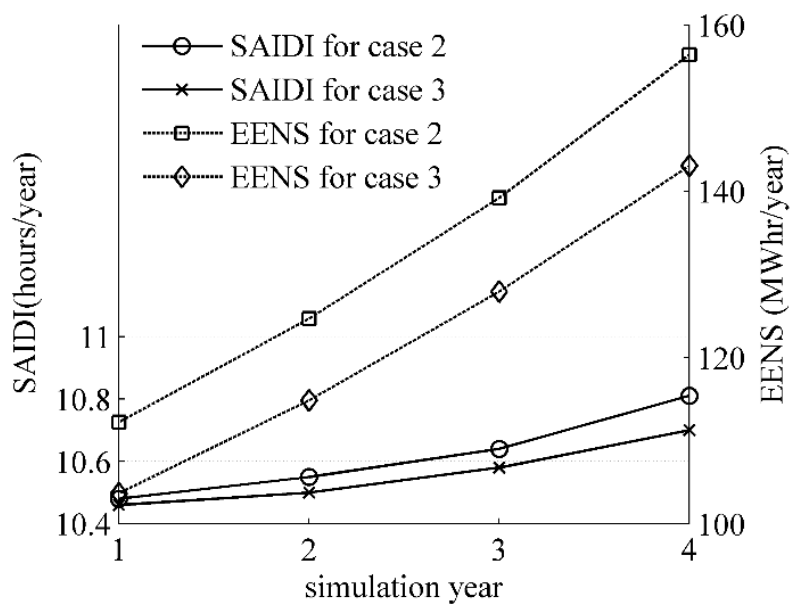

Figure 8. SAIDI and EENS for different years

\section{B. Impact of DGs on reliability}

Scenario 2 is considered for increment of reserve capacity. DGs are used to increase the system reserve capacity to increase the reliability and satisfy the future load demand on the system. In the following section results are compared with scenario 1 . If load growth is not considered and system reserve capacity is considered as $20 \%$ then the EENS values are 106.08 MWhr/year and 94.17 MWhr/year for scenario 1 and scenario 2 respectively, thus implying a good reliability of DG integration (scenario 2) as compared to scenario 1 . This is explained as follows, in scenario 1 due to the up gradation of substation and feeders' capacity, load transfer capability of feeders is increased. During the failure of any feeder section, the downstream load points are restored using alternate feeder provided there is an interconnection point between alternative feeder and downstream load points. Thus, if this constraint is not satisfied, then the restoration of loads will fail.

In case of scenario 2, DG integration improves both the feeder load transfer capability and islanding capacity of feeders. This will effectively restore more load points during the system failures. System load growth rate is considered as $10 \%$ and sequential load growth model was used to find the reserve capacity impact on reliability under two scenarios. System reliability for different reserve capacities is shown in Figure 9 for both the scenarios. It is observed that SAIDI and EENS are improved noticeably in scenario 2. There is no considerable change in reliability beyond the $40 \%$ reserve capacity.

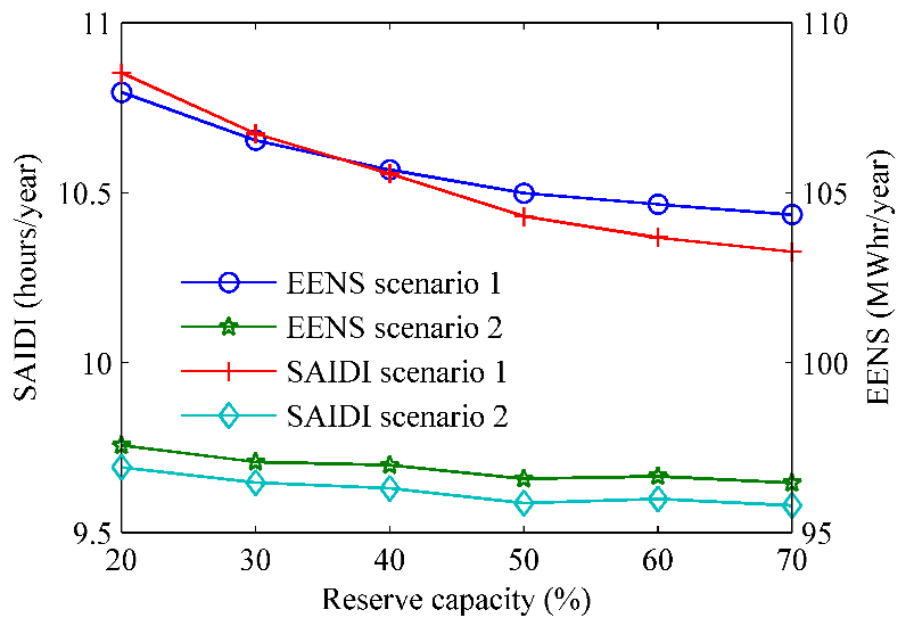

Figure 9. SAIDI and EENS for both scenarios 


\section{System capacity expansion planning}

In this section, system capacity expansion planning is discussed in terms of required reserve capacity and required time for capacity expansion considering the system load growth. A new measure $M A R C$ is used for system capacity expansion planning. Two different $M A R C$ values are considered for analysis to evaluate the impact of $M A R C$ on system capacity planning. Firstly, the $M A R C$ value is taken as zero for planning analysis. It means the system is allowed to operate up to zero reserve capacity and then it will go for capacity expansion. The final load on the system may not be the expected value due to the uncertainty nature of the system load growth rate as reliability concern, it is not possible to operate the system up to zero reserve capacity.

From the previous analysis of reserve capacity impact on system reliability (section 5.1), 20\% system reserve capacity is considered as minimum required reserve capacity. So MARC is taken as $20 \%$ for capacity expansion for better reliability. The estimation of required reserve capacity is given in Table 4 for different load growth rates and MARC values. The time for capacity expansion is considered as 3 years. The required reserve capacity is observed to increase with the increase of the load growth rate. It means that the system having higher load growth rates need to keep more reserve capacity at initial stage. It is also observed that the system required reserve capacity is increasing with $M A R C$ values.

Table 4. Estimation of reserve capacity and time for expansion

\begin{tabular}{ccccc}
\hline Growth rate & \multicolumn{2}{c}{ Reserve capacity $(\%)$} & \multicolumn{2}{c}{ Time for expansion } \\
\cline { 2 - 5 }$(\%)$ & $M A R C=20 \%$ & $M A R C=0 \%$ & $M A R C=20 \%$ & $M A R C=0 \%$ \\
\hline $5 \%$ & 38.91 & 15.76 & 5 years, 11 months & 9 years, 8 months \\
$10 \%$ & 59.72 & 33.1 & 3 years & 4 years, 11 months \\
$15 \%$ & 82.5 & 52 & 2years, 1 month & 3 years, 4 months \\
$20 \%$ & 107.36 & 72.8 & 1 year, 7 months & 2 years, 7 months \\
\hline
\end{tabular}

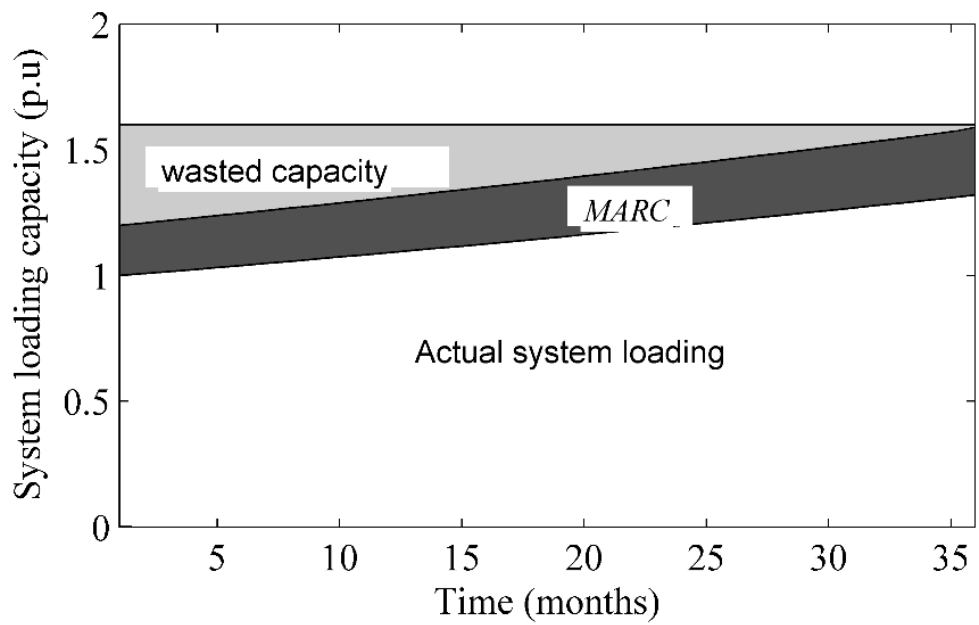

Figure 10. Constant system capacity planning

The unexpected conditions of the system loading and load growth leads to the system capacity expansion at any intermediate stage apart from the periodic system capacity expansion. The unexpected conditions consist of commercialization, industrialization and fast urbanization of distribution system serviceable area. The estimation of required time for capacity expansion is given in Table 4 for different system load growth rates and MARC values. System reserve capacity is considered as $60 \%$ at the instant of estimation of required time for capacity expansion. It can be seen that the required time margin is reducing with the increase of system load growth rate. The higher value of $M A R C$ causes the lesser time margin for system capacity expansion. 
The system capacity expansion planning is initiated after identifying the system load growth rate. From the Table 4, if distribution system experiences $10 \%$ load growth rate then system requires $60 \%$ reserve capacity for reliable operation of next 3 years with MARC value as $20 \%$. The installation of reserve capacity is performed in two ways. The total reserve capacity is installed at initial stage of system capacity expansion planning and it is shown in Figure 10. From the analysis of reserve capacity impact on system reliability, it is identified that the excess reserve capacity does not give more reliability benefits, instead it will increase the capital investment of the system. Most of the reserve capacity is wasted in this method during the initial months as shown in Figure 10.

A stepwise capacity expansion method is used to extract the maximum economic benefits from the system capacity expansion planning. The graphical illustration of this method is shown in Figure 11. Initially $40 \%$ reserve capacity $(M A R C=20 \%$ and $R C=20 \%)$ is installed instead of $60 \%$ reserve capacity, because the improvement of system reliability, in case of reserve capacity being increased from $40 \%$ to $60 \%$, is negligible, the system reserve capacity reaches to its MARC value after 21 months from the first system capacity expansion. So, after 21 months, the remaining $20 \%$ capacity is installed to get the reliability benefits. In this method, less reserve capacity is useless as shown in Figure 11.

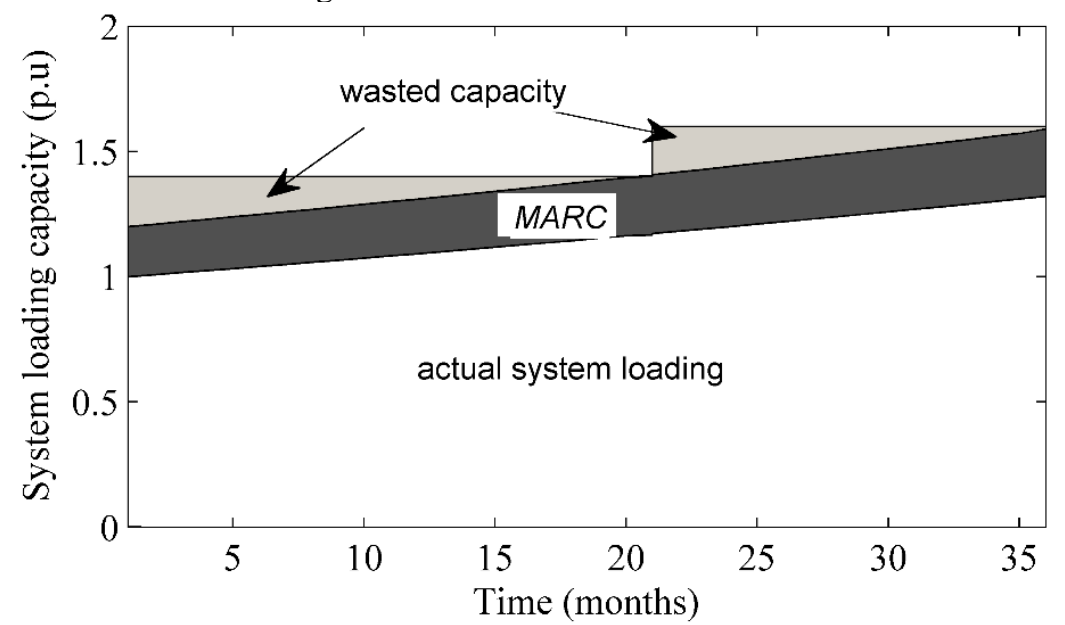

Figure 11. Step wise system capacity planning

\section{Conclusion}

In this paper, a sequential load growth model and capacity expansion model are proposed and are applied to an Indian distribution system. The sequential load growth model gives more realistic addition of load to the system due to load growth. The system reliability is evaluated using proposed model and compared with existing growth model for different load growth rates and reserve capacities and impact on reliability is analysed. The proposed load growth model gives realistic estimation of system reliability than existing model. The reliability is improving with the increase of reserve capacity, on the other hand, reliability is reducing with the increase of load growth rate. The relative change of reliability improvement is reduced with the increase of reserve capacity to higher levels. It is also observed that the required reserve capacity is increasing with the increase of load growth rate. The impact of load growth is reduced at high reserve capacity levels while using the proposed model. The reliability is reduced with the increase of time under load growth condition. From the impact analysis, it is possible to estimate the minimum required reserve capacity for a particular load growth rate for maximum reliability benefits along with lower investment. Two different scenarios are considered for increase of system reserve capacity. System reserve capacity's impact on reliability is studied for both scenarios, scenario 2 (DG integration) gives better reliability than the scenario 1(system capacity up gradation). The reserve capacity is estimated by including the MARC to reduce the risk due 
to load growth rate uncertainty. For a fixed duration of system capacity planning, the required reserve capacity is directly proportional to the load growth rate and required reserve capacity is found to change with the change of MARC value. At any instant, time required for next system capacity expansion is reduced with the increase of the load growth rate and MARC value. The step wise installation of the required reserve capacity is observed to reduce the wastage of reserve capacity thus reducing the capital investment.

\section{References}

[1]. Brown, R.E.; Burke, J.J., "Managing the risk of performance based rates," IEEE Trans. Power Syst., vol.15, no.2, pp. 893-898, May 2000.

[2]. Allan, R.N.; Billinton, R.; Sjarief, I.; Goel, L.; So, K.S., "A reliability test system for educational purposes-basic distribution system data and results," , IEEE Trans. Power Syst., vol.6, no.2, pp. 813-820, May 1991.

[3]. Martinez-Velasco, J.A.; Guerra, G., "Parallel Monte Carlo approach for distribution reliability assessment," IET Generat. Transm. Distrib., vol.8, no.11, pp. 1810-1819, November 2014.

[4]. Leite da Silva, A.M.; Nascimento, L.C.; da Rosa, M.A.; Issicaba, D.; Peças Lopes, J.A., "Distributed Energy Resources Impact on Distribution System Reliability Under Load Transfer Restrictions," IEEE Trans. Smart Grid., vol.3, no.4, pp. 2048-2055, December 2012.

[5]. Stefania Conti, and Santi Agatino Rizzo, "An Algorithm for Reliability Assessment of Distribution Systems in Presence of Distributed Generators", International Journal on Electrical Engineering and Informatics, vol. 7, no. 3, pp. 502-516, September 2015.

[6]. Ahmed S. A. Awad,Tarek H. M. El-Fouly, Magdy M. A. Salama "Optimal Distributed Generation Allocation and Load Shedding for Improving Distribution System Reliability" Elect. Power Compon. Syst., vol.42, no.6, pp. 576-584, March 2014.

[7]. Sankarakrishnan, A.; Billinton, R., "Sequential Monte Carlo simulation for composite power system reliability analysis with time varying loads," IEEE Trans. Power Syst., vol.10, no.3, pp. 1540-1545, July 1995.

[8]. A. M. Leite da Silva, L. A. Da Fonseca Manso, J. C. De Oliveira Mello and R. Billinton, "Pseudo-chronological simulation for composite reliability analysis with time varying loads," IEEE Trans. Power Syst., vol. 15, no. 1, pp. 73-80, Feb 2000.

[9]. Khyati D. Mistry, Ranjit Roy, "Enhancement of loading capacity of distribution system through distributed generator placement considering techno-economic benefits with load growth", Int J Electr Power Energy Syst, vol.54, pp. 505-515, January 2014.

[10]. Wadhwa, D.; Kumar, A., "Static Series Voltage Regulator in radial distribution system and impact of load growth and load models," RAECS 2014, Conf. Recent Advances in Engineering and Computational Sciences, Panjab University Chandigarh, India, pp. 1-8, March 2014.

[11]. K. Nagaraju, S. Sivanagaraju, T. Ramana and P. V. Prasad "A Novel Load Flow Method for Radial Distribution Systems for Realistic Loads" Elect. Power Compon. Syst., vol. 39, no.2, pp. 128-141, February 2011.

[12]. Chenghong Gu; Furong Li, "Long-run incremental cost pricing considering uncertain future load growth," IEEE Power \& Energy Society General Meeting, pp. 1-5, July 2009.

[13]. C. Gu, W. Yang, Y. Song, F. Li, "Distribution Network Pricing for Uncertain Load Growth Using Fuzzy Set Theory," IEEE Trans. Smart Grid, vol. 7, no. 4, pp. 1932-1940, July 2016.

[14]. Z. Wang, S. Wu, W. Liu, W. Ding, "Total supply capability for city distribution systems based on the probability load growth," 2014 IEEE Innovative Smart Grid Technologies Asia (ISGT ASIA), Kuala Lumpur, 2014, pp. 354-359.

[15]. S. S. Tanwar, D. K. Khatod, "A review on distribution network expansion planning," 2015 Annual IEEE India Conference (INDICON), New Delhi, 2015, pp. 1-6. 
[16]. S. S. Halilčević, A. F. Gubina and I. I. Softić, "The generation capacity reserve forecasting in power system," 2012 9th International Conference on the European Energy Market, Florence, 2012, pp. 1-8.

[17]. Z. Wang, Lihan Qin, C. Gu and F. Li, "Distributed storage capacity reservations for residential PV generation utilization and LV network operation," 2015 IEEE Power \& Energy Society General Meeting, Denver, CO, 2015, pp. 1-5.

[18]. J. P. Pfeifenberger, K. Carden, "Resource Adequacy Requirements: Reliability and Economic Implications", September 2013. Available:http://www.ferc.gov/legal/staffreports/2014/02-07-14-consultant-report.pdf

[19]. R. Billinton, R. N. Alan. Reliability Evaluation of Power Systems; 2nd Edition, Plenum Press; New York, 1995.

[20]. Roy Billinton, Wenyuan Li. Reliability Assessment of Electric Power Systems Using Monte Carlo Methods; $1^{\text {st }}$ Edition, Springer; 1994.

[21]. Peng Wang; Billinton, R., "Time sequential distribution system reliability worth analysis considering time varying load and cost models," IEEE Trans. Power Deliv., vol.14, no.3, pp. 1046-1051, August 1999.

[22]. Ke-yan Liu, Wanxing Sheng, Lijuan Hu, Yongmei Liu, XiaoliMeng, DongliJia, "Simplified probabilistic voltage stability evaluation considering variable renewable distributed generation in distribution systems," IET Generat. Transm. Distrib., vol. 9, no.12, pp. 1464-1473, August 2015.

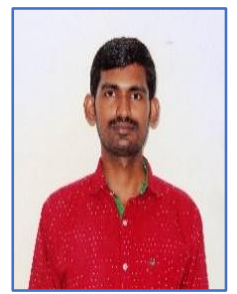

Galiveeti Hemakumar Reddy was born in Kadapa, Andhra Pradesh (India) in 1989. He received his B.Tech degree in electrical and electronics engineering and M.Tech degree in electrical power systems from Madanapalle Institute of Technology and Science, Madanapalle, Andhra Pradesh, India in 2010 and 2012 respectively. He is currently working toward the Ph.D. degree in electrical engineering, National Institute of Technology Silchar, Silchar, Assam, India.

His current research interests includes distribution system reliability, power quality assessment and mitigation, damage assessment, electric vehicles integration, distribution system restoration, fuzzy application in power systems.

Mr. Reddy is a student member of IEEE, IEEE PES society, IEEE smart grid society. He is a frequent reviewer of IEEE Transactions on Smart Grid, IEEE Transactions on Reliability and Electrical Power System Research.

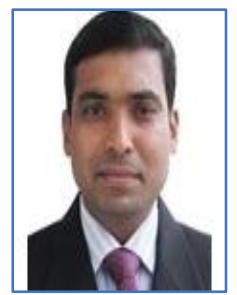

Arup Kumar Goswami was born in Midnapore West Bengal (India). He obtained his Bachelor of Technology in Electrical Engineering from Regional Engineering College, Kurukshetra (now, NIT Kurukshetra) in 1997. He obtained his Master of Engineering in Electrical and Electronics Engineering with Power Systems specializations in 2005 and PhD in 2010 from BIT Mesra and Indian Institute of Technology Roorkee respectively. Currently he is Assistant Professor in Electrical Engineering Department of National Institute of Technology Silchar, India.

His current research includes Power Quality, Power System Planning, Reliability, Congestion Management, Smart Grid, and Energy Management.

Dr. Goswami is a member of IEEE, life member of IE (I), life member of SESI, life member of CBIP, Member of IAENG and Member of IACSIT. He is a continuous reviewer of IEEE Transactions on Power Delivery, IET Generation Transmission and Distribution, Electric Power Components and Systems, International Journal of Electrical Power and Energy System, Electrical power system and research, European Transactions on Electric Power etc. 


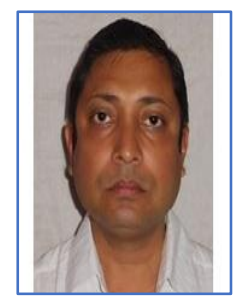

N. B. Dev Choudhury was born in Patacharkuchi, Assam in 1973. He obtained his B. E. in Electrical Engineering from Assam Engineering College under Gauhati University in 1994. He completed his masters from IIT Kanpur in 2003 and also completed PhD in 2011 from Jadavpur University, Kolkata, India. Currently he is Associate Professor in Electrical Engineering Department of National Institute of Technology Silchar, India.

His current reach interests includes deregulated power system, power economics, smart grid and reliability. He has more than 25 publications in various international referred journals and conferences.

Dr. Choudhury is a member of IEEE, IAENG and IACSIT. He is a continuous reviewer of many journals from IEEE, IET, Springer, Taylor \& Francis and Elsevier. 